\title{
Investigation into Time Response of Polymer Fiber Bragg Grating Based Humidity Sensors
}

\author{
W. Zhang, Member, IEEE, D. J. Webb, Member, IEEE, and G.-D. Peng,
}

\begin{abstract}
In this work we experimentally investigate the response time of humidity sensors based on polymer optical fiber Bragg gratings. By the use of etching with acetone we can control the poly (methyl methacrylate) based fiber in order to reduce the diffusion time of water into the polymer and hence speed up the relative wavelength change caused by humidity variations. A much improved response time of $\mathbf{1 2}$ minutes for humidity decrease and 7 minutes for humidity increase, has been achieved by using a polymer optical fiber Bragg grating with a reduced diameter of 135 microns.
\end{abstract}

Index Terms - Optical Polymers, Optical fiber devices, Fiber Bragg grating, Humidity measurement.

\section{INTRODUCTION}

$\mathrm{P}$ olymer optical fiber (POF) has been available for several decades. Its use has been overshadowed in the last two decades by the success of silica optical fiber. However, despite its high propagation loss, it is widely used in short-haul communication links due to its ease of use. Although recent technological advances have helped promote POF as a lower cost alternative to glass fiber or copper at short distances and bit rates of up to $10 \mathrm{Gbps}$ [1] the physical and chemical properties of polymeric materials are rather different to silica, potentially making it also attractive for researchers to exploit in device and sensing applications.

The main differences are:

- Mechanical properties: Compared to silica optical fibers, POF has a much higher failure strain $[2,3]$ and a Young's modulus approximately 25 times lower [4].

- Chemical properties: Polymer fibers can be modified using organic chemical techniques in order to improve the properties of the material.

- Thermal properties: POF Bragg gratings based on

Manuscript received June 30, 2011

W. Zhang is with the Photonics Research Group, Aston University, Birmingham B4 7ET, UK. (Telephone: 44-121-2043549; fax: 44-121-2043682; e-mail: w.zhang@ aston.ac.uk).

D. J. Webb is with the Photonics Research Group, Aston University, Birmingham B4 7ET, UK. (e-mail: d.j.webb@aston.ac.uk).

G.-D. Peng is with the School of Electrical Engineering and Telecommunications, University of New South Wales, Sydney, NSW 2052, Australia.

Copyright (c) 2011 IEEE. Personal use of this material is permitted. However, permission to use this material for any other purposes must be obtained from the IEEE by sending a request to pubs-permissions@ieee.org. poly(methyl methacrylate) (PMMA) have approximately four times the thermal sensitivity of silica gratings [5].

- Biocompatibility: Polymer fiber may be used for in vivo applications where the use of glass is inappropriate due to danger from breakages.

More recently, fiber Bragg gratings (FBGs) have been inscribed into step index [2] and microstructured POF [6] based on PMMA. Polymer optical fiber Bragg gratings (POFBGs) in such fiber were found to be sensitive to relative humidity, temperature and strain. This is in contrast to glass fiber FBGs which do not show any intrinsic humidity sensitivity. The affinity for water of PMMA, leads to a swelling of the fiber and an increase of refractive index, both of which contribute to an increase in the Bragg wavelength of a FBG written in the fiber [7]. This is a potentially very useful property, which has possible applications in chemical processing, agriculture, food storage, paper manufacturing, semiconductor and pharmaceutical industries, where humidity is monitored and controlled to ensure product quality. POFBGs have recently been applied, for example, to quantifying the small amount of water present in aviation fuel [8].

There have been many optical fiber humidity sensors reported. Among them some techniques (e.g., [9-11]) are mainly absorption and colorimetric-based methods, which rely on the reaction of moisture-sensitive chemical reagents immobilized in a suitable support matrix. Methods of fabrication for these sensors are expensive. Those sensing operations rely on chemical reactions which are irreversible and have a limited shelf life. Humidity introduces photoelastic refractive index change in some polymer materials. It leads to another group of optical fiber humidity sensors in which the humidity related refractive index changes are detected by measuring different parameters like evanescent field related light transmission [12, 13], surface plasmon resonance [14], cladding mode coupling [15], interferometric phase [16], etc.. In these sensors the polymers are coated onto different optical fiber/waveguide structures in a thin layer (usually 10s of nanometers) to produce fast response time. The main drawbacks are their susceptibility to contamination, the presence of interference.

In POFBG humidity sensors the water affinity of the fiber introduces significant Bragg wavelength change. Therefore the sensors are wavelength coded, immune to the variations in light source and transmission loss. The POFBGs need no extra coating or special waveguide structure for humidity sensing, so 
they are compact, robust, and cost effective. However, the water absorption process is a slow one. When POFBGs are applied to humidity sensing, the response time is a key issue, which is particularly important for the applications where accurate humidity control is required. In this work we investigate the time responses of POFBG based humidity sensors made of different polymer fibers with different fiber geometries. A much improved time response is achieved by etching the fiber using acetone to reduce the fiber diameter.

\section{OPERATION PRINCIPLE}

PMMA and many other polymers have the tendency to absorb water [17, 18]. PMMA exhibits a maximum water content of up to $2 \mathrm{wt} \%$ [19]. Many polymers show water absorption processes, where clustering of water occurs within the polymer matrix leading to an absorption increase at higher humidity. This process leads to a non linear relation of fiber water content as a function of relative humidity surrounding the fiber [20], which has been used to enable humidity sensing with optical fiber sensors by use of either a polymer coated silica fiber [e.g., 21] or directly using polymer optical fiber [8].

The Bragg wavelength depends on the effective core index, $n_{\text {eff }}$, and the grating pitch, $\Lambda$ which both depend on the temperature $T$ and the water content $w$ :

$$
\lambda_{\text {Bragg }}=2 n_{\text {eff }}(T, w) \Lambda(T, w)
$$

For constant temperature the Bragg wavelength change shows a non-linear dependence on the relative humidity $(\mathrm{RH})[7$, 20]. Following references [7, 20] we assume that the Bragg wavelength shifts linearly with water content in the fiber, and the water content in the polymer, $w$, is introducing the non-linearity. The non-linear water content dependence on the relative humidity, $H$, is [7]

$$
w / w_{\max }=w_{n}=H^{m}
$$

where $w_{\max }$ is the maximum water content, $m$ represents the non-linearity. Therefore the Bragg wavelength can then be expressed in terms of the relative humidity as

$$
\lambda_{B}=\lambda_{B o}+c H^{m}
$$

where $\lambda_{B 0}$, is the initial Bragg wavelength, $c$, a coefficient associated with PMMA swelling, humidity dependence of the refractive index, and swelling induced stress.

A sudden change of environmental condition could lead to the water content change in the PMMA, which is a function of time. This process of water absorption or desorption in PMMA can be described by the diffusion theory of mass transfer. For a cylinder system, if the surface concentration, $C_{0}$, is constant and the concentration, $C_{1}$, is initially uniform throughout the cylinder, then the mass concentration due to diffusion can be expressed as [22]

$$
\frac{C-C_{1}}{C_{0}-C_{1}}=1-\frac{2}{a} \sum_{n=1}^{\infty} \frac{\exp \left(-D \alpha_{n}^{2} t\right) J_{0}\left(r \alpha_{n}\right)}{\alpha_{n} J_{1}\left(a \alpha_{n}\right)}
$$

where $D$ is diffusion coefficient, $t$ the time, $r$ the radial position, $a$ the cylinder radius; $\alpha_{\mathrm{n}}$ is the $n$th positive root of

$$
J_{0}\left(a \alpha_{n}\right)=0
$$

Equation (4) can be used to define the water concentration in the polymer fiber for either water in-diffusion (absorption) or out-diffusion (desorption). However, the diffusion coefficient for in-diffusion and out-diffusion is different. Usually in PMMA the water desorption is faster than water absorption, which means a larger diffusion coefficient for out-diffusion [7, 23]. In (4) only the diffusion coefficient and the fiber radius can be varied to accelerate or decelerate the diffusion process. In terms of normalized concentration the equation (4) can be rewritten as

$$
C(t, r)=1-\frac{2}{a} \sum_{n=1}^{\infty} \frac{\exp \left(-D \alpha_{n}^{2} t\right) J_{0}\left(r \alpha_{n}\right)}{\alpha_{n} J_{1}\left(a \alpha_{n}\right)}
$$

for in-diffusion, or

$$
C(t, r)=\frac{2}{a} \sum_{n=1}^{\infty} \frac{\exp \left(-D \alpha_{n}^{2} t\right) J_{0}\left(r \alpha_{n}\right)}{\alpha_{n} J_{1}\left(a \alpha_{n}\right)}
$$

for out-diffusion.

\section{EXPERIMENTS AND RESULTS}

In the reported polymer optical fiber humidity sensors (including POFBGs and polymer coated silica FBGs) the response time ranges from $25 \mathrm{~min}$ (coating thickness of $33 \mu \mathrm{m}$ ) [21] to $80 \mathrm{~min}$ (coating thickness of $29 \mu \mathrm{m}$ ) [24]. In reference [8] a response time of $30 \mathrm{~min}$ was reported when using a POFBG with a fiber diameter of $195 \mu \mathrm{m}$ while a response time of one hour was measured for a POFBG with a diameter of 125 $\mu \mathrm{m}$ in [7]. The swelling of polymer optical fiber dominates the wavelength change of the POFBG and the main influence on the humidity time response comes from the diffusion coefficient for water into the PMMA, and the geometry of the POF.

We therefore carried out a series of experiments to investigate this influence on the humidity time response of POFBG sensors by measuring the response time for Bragg gratings written in different polymer optical fibers with different diameters.

POFBGs were fabricated by attaching a $10 \mathrm{~cm}$ length of POF to a single mode silica fiber down-lead using UV curable glue (Norland 76). The PMMA based POF contained a $5 \mathrm{~mm}$ long FBG, fabricated by illuminating from above a phase mask placed on top of the POF using 325nm UV light from a $\mathrm{HeCd}$ laser.

For testing, the POFBG sensors were placed inside an environmental chamber (Sanyo Gallenkamp). They were illuminated via a circulator with light from a broadband light source and observed in reflection using an IBSEN I-MON 400 wavelength interrogation system, see Fig.1. Relative humidity is defined as the ratio of the amount of water vapor present in the atmosphere to the maximum amount that the atmosphere can hold at the existing temperature. The environmental chamber was set to different relative humidities while the temperature was fixed. The POFBG sensors are sensitive to both humidity and temperature. In practical application this cross sensitivity of the sensor should be considered and eliminated. This can be 
realized by using a dual parameter sensor in which a second FBG is inscribed in silica fiber down-lead [25]. Since the silica FBG exhibits no intrinsic humidity sensitivity (in [25] a recoated silica FBG showed a humidity sensitivity 125 times lower than that of the POFBG used), a well-conditioned response can be achieved.

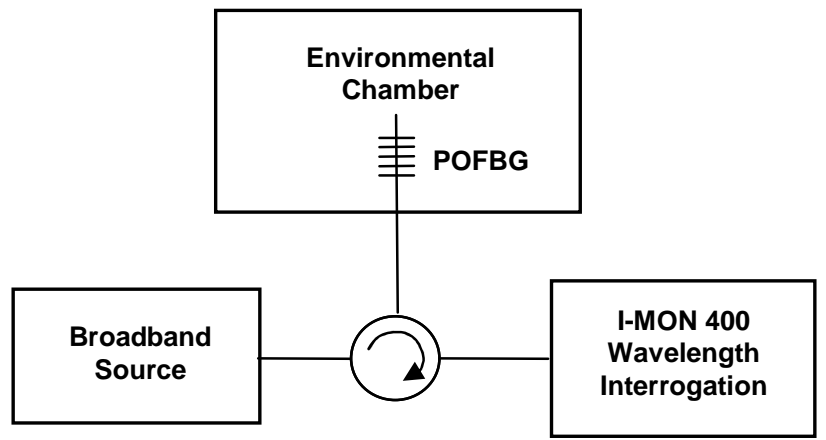

Fig. 1. Experimental arrangement

\section{A. Different polymer fibers}

Three different POF based FBGs were placed in the chamber for testing. The cladding of all the fibers was formed from a mixture of methyl and ethyl methacrylate, while the cores contained methyl, ethyl and benzyl methacrylate, plus a small amount of photosensitizer. The measured humidity responses are shown in Fig. 2 where the relative humidity $(\mathrm{RH})$ was step-changed from $80 \%$ to $70 \%$ while the chamber temperature was kept at $25^{\circ} \mathrm{C}$. To simplify comparison of the sensors which have different Bragg wavelengths, we chose to plot the time response of the relative wavelength change, $\Delta \lambda / \Delta \lambda_{\max }$, which is the ratio of the grating's Bragg wavelength deviation from its original value to the maximum wavelength deviation induced by the relative humidity change. The core and cladding diameters of POF1, POF2 and POF3 were originally $8 \mu \mathrm{m} \& 150 \mu \mathrm{m}, 8$ $\mu \mathrm{m} \& 190 \mu \mathrm{m}$, and $7 \mu \mathrm{m} \& 190 \mu \mathrm{m}$, respectively while their respective Bragg wavelengths at $25^{\circ} \mathrm{C}$ and $70 \% \mathrm{RH}$ were $1571.4 \mathrm{~nm}, 1568.7 \mathrm{~nm}$ and $1535.2 \mathrm{~nm}$.

The measured POFBG humidity responses and temporal responses of normalized water concentration in the fiber core for these 3 polymer optical fibers, calculated using Eq. (5B) are illustrated in Fig. 2 (the diffusion coefficient is taken as a free parameter and adjusted to provide the best fit to the data). From Fig. 2 one can notice that for POF1 and POF2 the relative Bragg wavelength change is not stabilized 1 hour after the relative humidity step change. The response time then was estimated as the time of relative wavelength change being reduced to $10 \%$, with the help of an exponential fit to the experimental data. From this the response times for POF1, POF2 and POF3 were estimated as $58 \mathrm{~min}, 62 \mathrm{~min}$ and $38 \mathrm{~min}$, respectively.

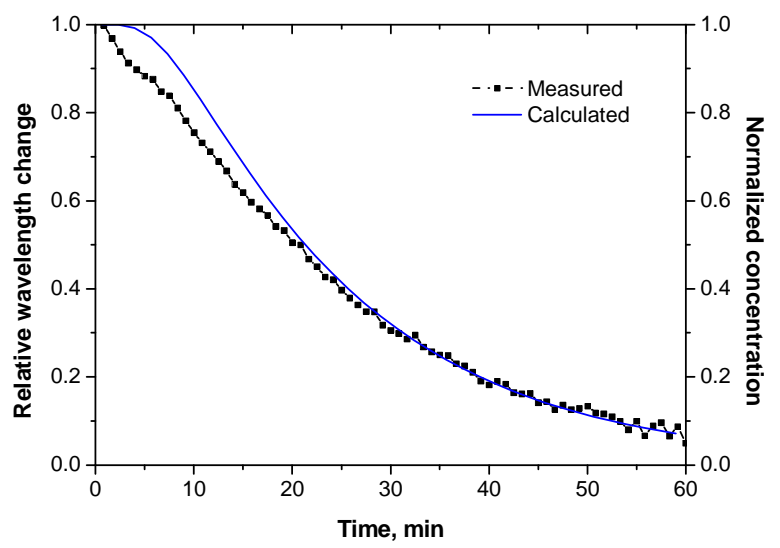

(a)

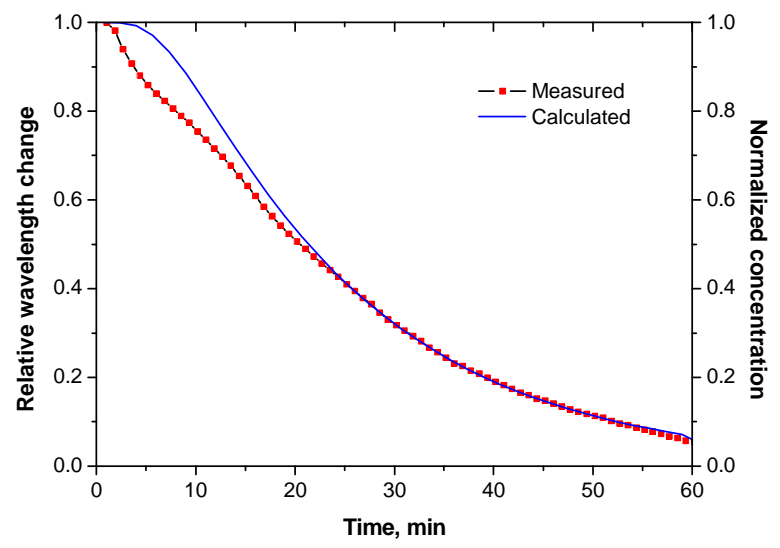

(b)

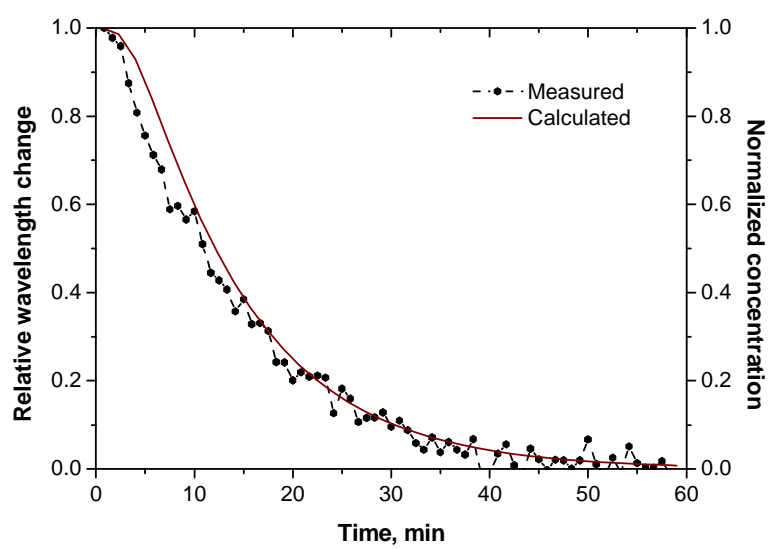

(c)

Fig. 2. Measured relative wavelength changes of 3 POFBG sensors and calculated temporal responses of normalized concentration in the core of 3 POFs. A step relative humidity change from $80 \%$ to $70 \%$ was applied. (a) POF1, (b) POF2, and (c) POF3.

A more accurate estimation of response time can be analytically calculated by using equation (5B) for out-diffusion. It generates the normalized water concentration change in the fibers (shown in Fig. 2), which is related to the relative wavelength changes of the sensors. The estimated response times now are $53 \mathrm{~min}, 51 \mathrm{~min}$ and $31 \mathrm{~min}$, respectively, for POF1, POF2 and POF3. 
From Fig. 2 the diffusion coefficient, $D$, can also be estimated. The diffusion coefficient for POF1 is $9.0 \times 10^{-9} \mathrm{~cm}^{2} / \mathrm{s}$, which is in good agreement with values for PMMA for desorption published in $[7,22]$. However, the diffusion coefficients for POF2 and POF3 are $13.5 \times 10^{-9} \mathrm{~cm}^{2} / \mathrm{s}$, and $23.0 \times 10^{-9} \mathrm{~cm}^{2} / \mathrm{s}$ respectively. The latter two fibers obviously show much higher diffusion coefficients, and exhibit a reasonable response time for even larger fiber diameter.

It should be pointed out that the calculated results are the water concentrations at fiber core, based on equations $(5 \mathrm{~A})$ and (5B). It takes some time for the water to reach the core. However, as soon as it enters the cladding there will be some expansion of the fiber and some wavelength change of the POFBG. It means that in the initial stage of the humidity change the wavelength change observed is faster than the calculated concentration change. This difference is clearly seen in Fig. 2.

\section{B. Etching of Polymer optical fibers}

PMMA can be easily dissolved in some chemical solvents, for example, acetone. Acetone is a self-associated solvent. As a consequence, it would develop preferentially self-associations (between solvent molecules) rather than PMMA/solvent interactions [26]. Hence the PMMA chains remaining as part of the fiber would not be affected by etching. This provides a simple and effective way to reduce the diameter of POF to a desired value. Acetone etching was applied to the aforementioned POFs. An Axioskop 2 MOT Plus optical microscope was used to inspect the fiber before and after the etching and estimate the fiber diameter reduction. Fig. 3 shows the microscopic images of a sample of POF3 before and after etching. From microscopic images there is no obvious change of the fiber surface other than the fiber diameter reduction.

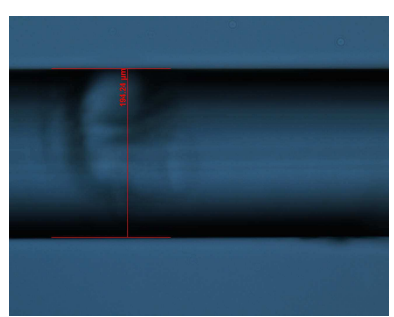

(a)

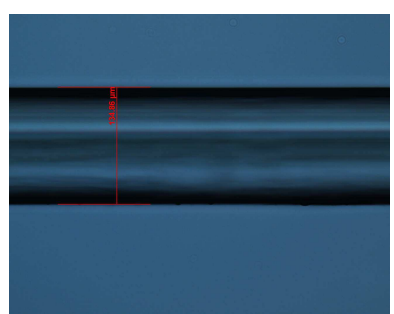

(b)
Fig. 3. The microscopic images of a POF. (a) before etching $(190 \mu \mathrm{m}$ in diameter), (b) after etching (135 $\mu \mathrm{m}$ in diameter).

The resulting diameter reductions by etching process are shown in Fig. 4.

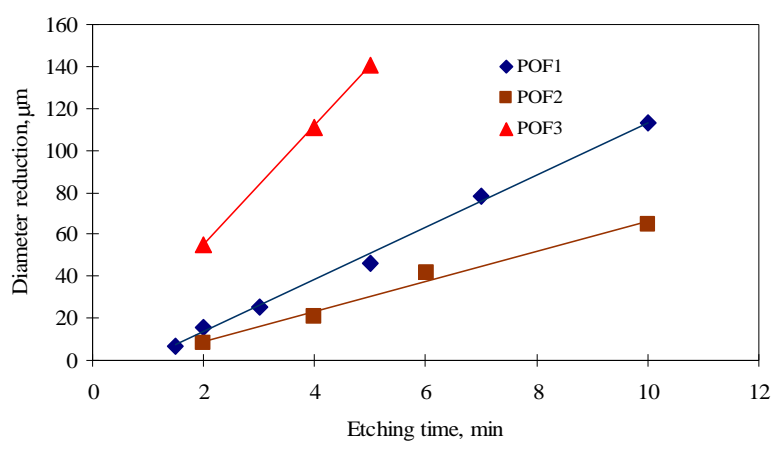

Fig. 4. POF diameter reduction due to acetone etching.

From Fig. 4 one can notice that POF3 shows the fastest etching rate and POF1 the slowest. Considering the results shown in Fig. 2 there seems to be a possible link between the response time and etching rate of the POF. POF3 shows the fastest etching rate and shortest response time and POF1 shows the slowest etching rate and a long response time though the fiber diameter of POF1 is much smaller than the other two. It seems that the high diffusion rate of water that leads to a short humidity response rate may be correlated with a fast diffusion of acetone into the PMMA permitting more rapid etching.

The cladding material of the three fibers was polymerized from the same mixture of methyl and ethyl methacrylate. The three fiber samples came from three different preforms and it is likely that the polymerisation did not proceed at the same rate in each case. We propose that this difference is probably responsible for the different behaviour of the samples.

\section{Polymer optical fibers with different diameters}

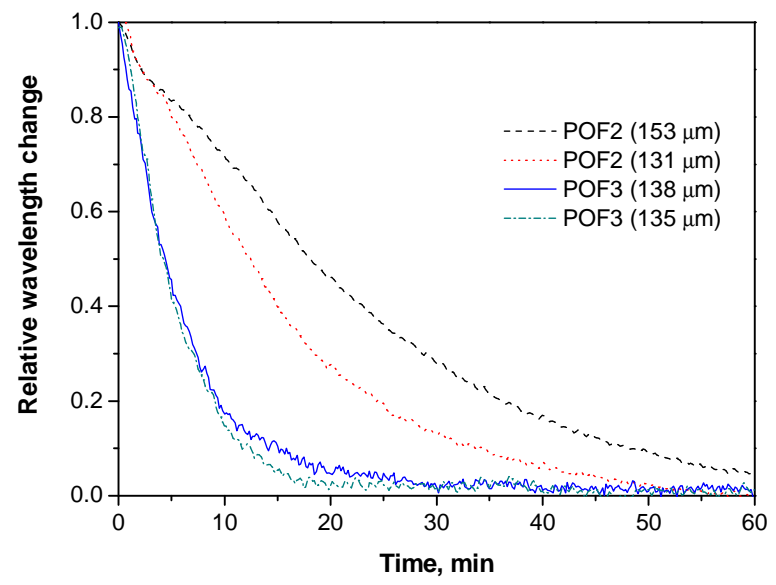

(a) 


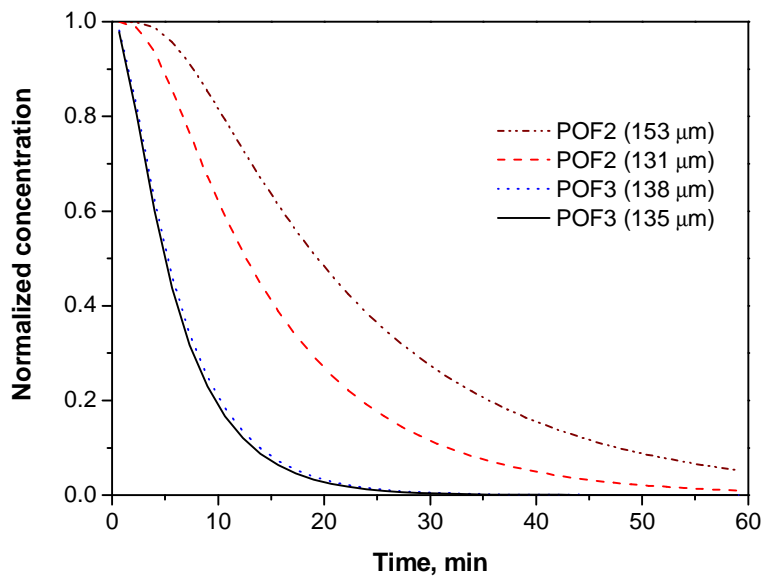

(b)

Fig. 5. Relative wavelength changes of POFBG sensors with different fiber diameters against a step relative humidity drop. (a) measured responses, (b) calculated responses.

We then etched several Bragg gratings written in POF2 and POF3. The etched POFBGs were placed in the environmental chamber to enable investigation of the time response to relative humidity. Fig. 5(a) shows some typical results when the environmental chamber was set to the same condition as used previously (relative humidity step change from $80 \%$ to $70 \%$, temperature set at $25^{\circ} \mathrm{C}$ ). Again the response time was estimated based on the calculated responses shown in Fig. 5(b). All the response times are summarized in Table 1.

TABLE 1

RH SENSING RESPONSE TIMES OF DIFFERENT POFS

\begin{tabular}{cc}
\hline \hline POFBG & Response time $^{\mathrm{a}}$ \\
\hline POF1 $(150 \mu \mathrm{m})$ & $53 \mathrm{~min}$ \\
POF2 $(190 \mu \mathrm{m})$ & $51 \mathrm{~min}$ \\
POF2 $(153 \mu \mathrm{m})$ & $48 \mathrm{~min}$ \\
POF2 $(131 \mu \mathrm{m})$ & $32 \mathrm{~min}$ \\
POF3 $(190 \mu \mathrm{m})$ & $31 \mathrm{~min}$ \\
POF3 $(138 \mu \mathrm{m})$ & $14 \mathrm{~min}$ \\
POF3 $(135 \mu \mathrm{m})$ & $12 \mathrm{~min}$
\end{tabular}

${ }^{a}$ The response time was calculated as the time of normalized concentration being reduced to $10 \%$.

The results listed in the table show a clear trend that for the same type of POF the response time for humidity sensing decreases with reduced fiber diameter. POF3 shows the best performance among the POFs used in this work in which the response time is greatly reduced to $12 \mathrm{~min}$. This is a satisfying speed for applications such as long term monitoring of water in fuel. Further reducing the diameter in POF3 could provide faster response time; however, it may compromise the ease of handling.
D. Responses of in-diffusion and out-diffusion

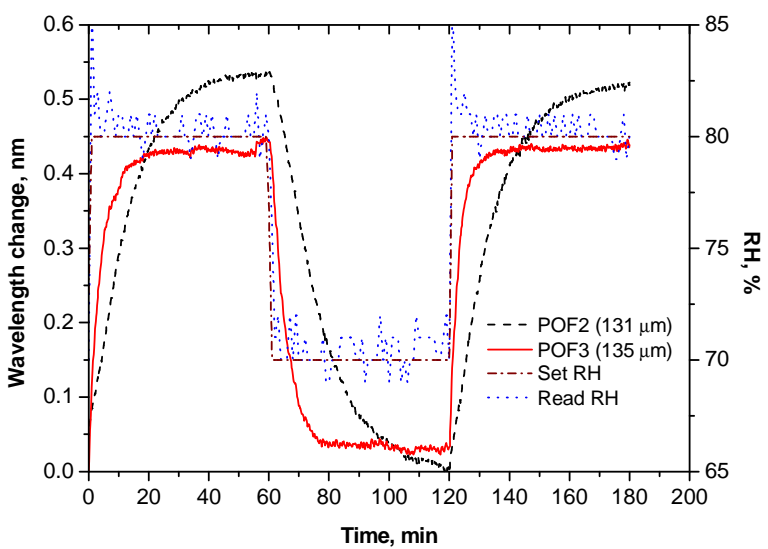

Fig. 6. Full cycle responses of two POFBG sensors. RH varied from $80 \%$ to $70 \%$ and back to $80 \%$ while the temperature was kept constant at $25^{\circ} \mathrm{C}$.

Fig. 6 shows the full cycle response for humidity sensing using POF2 $(131 \mu \mathrm{m})$ and POF3 $(135 \mu \mathrm{m})$ where RH varied from $80 \%$ to $70 \%$ and back to $80 \%$ while the temperature was kept constant at $25^{\circ} \mathrm{C}$. Each value of humidity was kept for one hour. As a comparison the set value and measured value from the environmental chamber's built-in RH sensor are also depicted in the figure. From the figure it can be seen that both the sensors followed the RH change. POF3 $(135 \mu \mathrm{m})$ shows a faster response more closely following the humidity change.

Based on the full cycle responses for humidity sensing the response times of these two sensors for both in-diffusion and out-diffusion have been estimated. The response times are estimated from the measured responses shown in Fig. 7, as the time of normalized concentration change being reduced to $10 \%$ for humidity step drop (out-diffusion), and the time of normalized concentration change being increased to $90 \%$ for humidity step rise (in-diffusion). The corresponding responses are calculated by using equations (5A) and (5B) and also shown in Fig. 7. The calculated response times are summarized in Table 2.

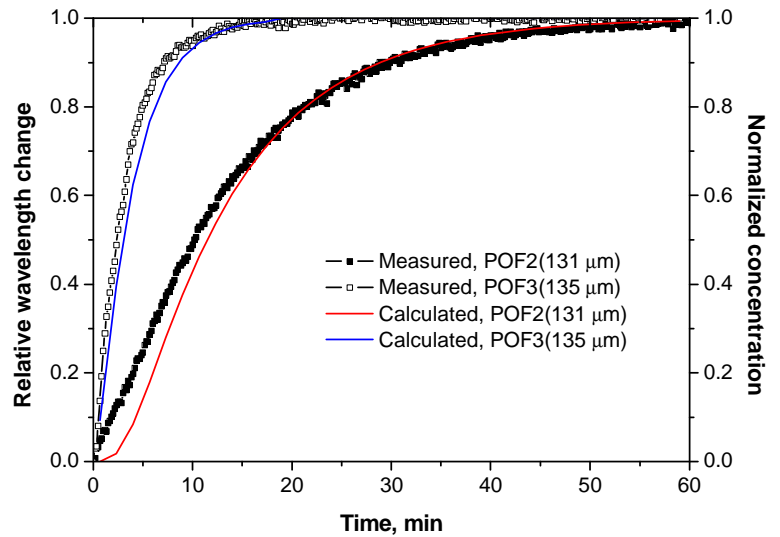

(a) 


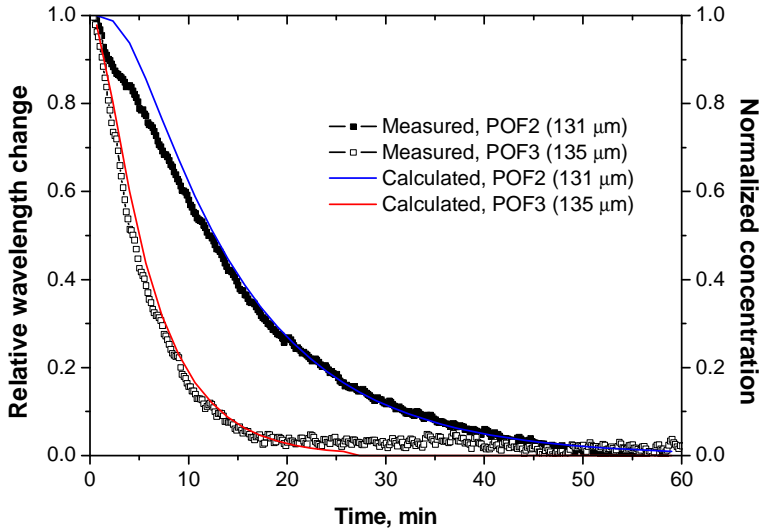

(b)

Fig. 7. Measured and calculated step humidity responses of two POFBG sensors for (a) in-diffusion and (b) out-diffusion.

TABLE 2

RESPONSE TIMES FOR IN- AND OUT-DIFFUSION

\begin{tabular}{ccc}
\hline POFBG & IN-DIFFUSION & OUT-DIFFUSION \\
\hline POF2 $(131 \mu \mathrm{m})$ & $29 \mathrm{~min}$ & $32 \mathrm{~min}$ \\
POF3 $(135 \mu \mathrm{m})$ & $7 \mathrm{~min}$ & $12 \mathrm{~min}$ \\
\hline \hline
\end{tabular}

\section{E. Dynamic response of POFBG sensor}

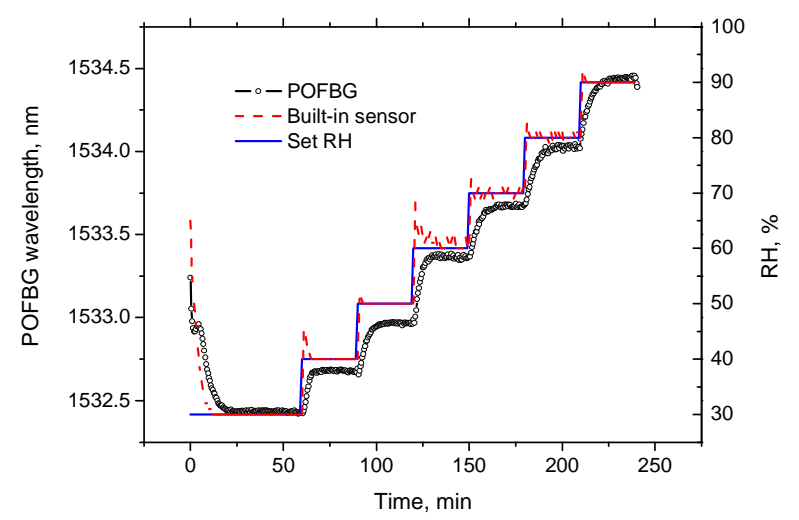

(a)

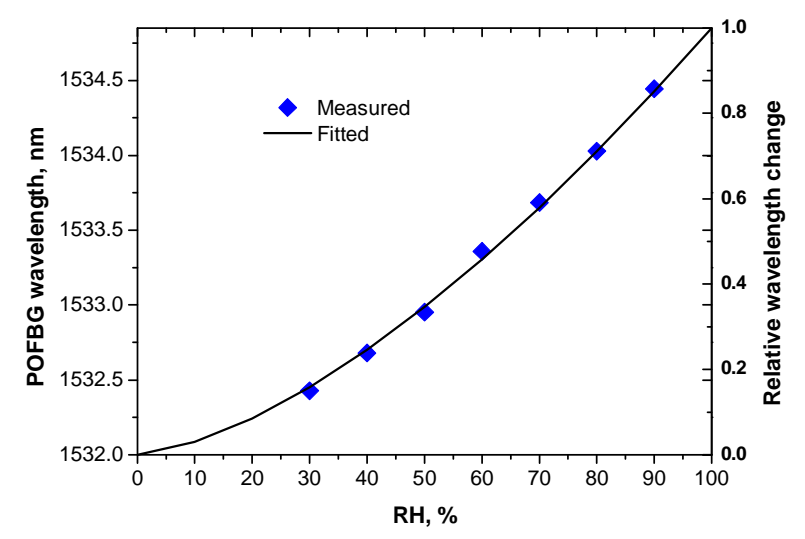

(b)
Fig. 8. Dynamic RH response of POF3 $(135 \mu \mathrm{m})$. (a) measured responses by PFBG and built-in sensor, and set value by programming, (b) POFBG wavelength vs. $\mathrm{RH}$, and the fitted relative wavelength change vs. $\mathrm{RH}$.

Further experiments were carried out to demonstrate the POFBG performance over a larger $\mathrm{RH}$ range. The environmental chamber was programmed to change the relative humidity from $30 \%$ to $90 \%$ with an increment of $10 \%$ every 30 min while the temperature was fixed at $25{ }^{\circ} \mathrm{C}$. A typical response of POF3 $(135 \mu \mathrm{m})$ is shown in Fig. 8a. The values of the set humidity and that measured by the chamber's built-in RH sensor are depicted in the figure as a comparison. From Fig. 8a one can see that the POFBG sensor follows the humidity change closely. The stabilized POFBG wavelength at each $\mathrm{RH}$ is depicted in Fig. 8b. It shows a slightly nonlinear response against RH. According to equations (2) and (3) the POFBG wavelength varies with $\mathrm{RH}$ in the form of $H^{m}$ where $m$ represents non-linearity. Regression analysis on the data generated a relation between the wavelength change of the POFBG and the relative humidity, as illustrated in Fig. 8b. It indicates a non-linearity, $m$, of 1.52 , which is close to the values reported (1.49 in [7], and 1.47 in [20]).

The average responsivity over $30 \%$ to $90 \%$ of $\mathrm{RH}$ is 33.6 $\mathrm{pm} / \%$. The minimum detectable $\mathrm{RH}$ was estimated based on the measured response for $50 \%$ of $\mathrm{RH}$ as the environmental chamber was best controlled at that value over 25 minutes. The standard deviation of the measured POFBG response over that period is $4 \mathrm{pm}$, which indicates a minimum detectable $\mathrm{RH}$ of $0.12 \%$.

\section{DisCUSSION AND CONCLUSION}

Since water diffusion in polymer optical fiber can be described by the diffusion theory of mass transfer the humidity response of POFBGs with known diffusion coefficient and geometry can be predicted. The response time can also be improved by choosing polymer optical fiber with an optimized diffusion coefficient or modifying the fiber geometry. The fibers used have different diffusion coefficients therefore different response times to humidity change. By acetone-etching the polymer optical fiber we can change the fiber diameter. Experimental results have shown much improved time response of POFBG humidity sensor with reduced fiber diameter.

The general performance of POFBG humidity sensing may be predicted and described by the equations established from diffusion theory. As mentioned earlier all the diffusion coefficients are estimated from the measurement results. We obtained the diffusion coefficients for all 3 fibers from the measured responses of POFBGs without being etched. We noticed, however, that for the POFBGs with reduced diameters (i.e., after etching) the diffusion coefficients are quite different. For the measured responses shown in Fig. 4(a) the corresponding calculations in Fig. 4(b) indicate that the diffusion coefficient for POF2 decreases to $10.5 \times 10^{-9} \mathrm{~cm}^{2} / \mathrm{s}$ from $13.5 \times 10^{-9} \mathrm{~cm}^{2} / \mathrm{s}$; the diffusion coefficient for POF3 increases to $25.5 \times 10^{-9} \mathrm{~cm}^{2} / \mathrm{s}$ from $23.0 \times 10^{-9} \mathrm{~cm}^{2} / \mathrm{s}$. The difference is too big to attribute to measurement error. We suggest this difference may be due to the polymerization of the 
original preform cladding proceeding at different rates with distance from the core, leading to a diffusion coefficient that varies with position.

According to the literature, water desorption in PMMA is faster than water absorption [23], i.e., the out-diffusion coefficient is larger than the in-diffusion coefficient. The results summarized in Table 2, however, show an opposite trend. Two of the sensors before (POF2 and POF3) exhibited the same trend. The origin of this contradiction is not very clear, however, the authors have one hypothesis. In the water absorption process, when water initially enters the fiber cladding but has not yet reached the core, the expansion of the cladding will nevertheless apply a detectable strain to the fiber core, resulting in a Bragg wavelength change. Therefore in the absorption process the POFBG wavelength increase takes a shorter time than predicted by the water concentration change in the fiber core.

The stability of POFBG performance is an issue. The grating strength and wavelength could vary over time due to relaxation of the frozen-in molecular alignment induced by the fiber drawing process. We have shown that annealing the fiber can permit this relaxation, leading to stable POFBG performance and an extended operating temperature range [27]. The latest research [28] suggests that long term stability of annealed POFBG wavelength and sensitivity can be expected as long as the device operates below a certain temperature threshold. In our work none of the POFBGs used showed significant change of performance over a span of six months.

In conclusion, we have experimentally investigated the influence of different polymer optical fibers with different diameters on the time response of humidity sensing. Controllable etching has been used to reduce the fiber diameter leading to a much improved humidity sensing response time of down to 7 minutes. The rather different responses of the three samples suggests that a detailed study of the influence of the polymerization process on the diffusion rate would be useful, possibly leading to an even faster response time. Based on this technique the response of POFBG humidity sensors can be improved to enhance performance for many potential applications.

\section{REFERENCES}

[1] G. Giaretta, W. White, M. Wegmuller, and T. Onishi, "High-speed (11 Gbit/s) data transmission using perfluorinated graded-index polymer optical fibers for short interconnects $(<100 \mathrm{~m})$," IEEE Photon. Tech. Lett., vol.11, pp. 347-349, 2000.

[2] Z. Xiong, , G. D. Peng, , B. Wu, and P. L Chu, "Highly tunable Bragg gratings in single-mode polymer optical fibers," IEEE Photon. Tech. Lett., vol.11, pp. 352-354, 1999.

[3] C. Emslie, "Review: Polymer optical fibers," J. Mater. Sci., vol. 23, pp 2281-2293, 1988.

[4] J. Brandrup, Polymer Handbook. Vol. 1\&2. Wiley. 1999.

[5] Webb, D.J. and K. Kalli, Polymer fibre Bragg gratings, in Fiber Bragg Grating Sensors: Thirty Years from Research to Market, A. Cusano, Editor. 2010, Bentham eBooks

[6] H. Dobb, D. J. Webb, K. Kalli, A. Argyros, M. C. J. Large, and M. A. van Eijkelenborg," Continuous wave ultraviolet light-induced fiber Bragg gratings in few- and single-mode microstructured polymer optical fibers," Opt. Lett., vol.30, pp. 3296-3298, 2005
[7] N. G. Harbach, "Fibre Bragg gratings in polymer optical fibres." $\mathrm{PhD}$ thesis Lausanne: EPFL, 2008

[8] C. Zhang, X. Chen, D. J. Webb, and G.-D. Peng, "Water detection in jet fuel using a polymer optical fibre Bragg grating", Postdeadline paper, 20th International Conference on Optical Fibre Sensors, 2009.

[9] M. F. Choi and O. L. Tse, "Humidity-sensitive optode membrane based on a fluorescent dye immobilized in gelatin," Anal. Chim. Acta., vol. 378, p. 127,1999 .

[10] A. P. Russell and K. S. Fletcher, "Optical sensor for the determination of moisture," Anal. Chim. Acta., vol. 170, p. 209, 1985.

[11] A. Kharaza and B.E. Jonesa, "A distributed optical-fibre sensing system for multi-point humidity measurement”, Sens. Actuators: A: Phys vol. 47, 1995, P. 491, 1995

[12] S. Muto, O. Suzuki, T. Amano, and M. Morisawa, "A plastic optical fibre sensor for real-time humidity monitoring," Meas. Sci. Technol., vol. 14, p. 746, 2003.

[13] L. Zhang1, F. Gu, J. Lou, X. Yin, and L. Tong, "Fast detection of humidity with a subwavelengthdiameter fiber taper coated with gelatin film", Opt. Express, vol. 16, p. 13349, 2008

[14] M. N. Weiss, R. Srivastava, and H. Groger, "Experimental investigation of a surface plasmon-based integrated-optic humidity sensor", Electron. Lett., vol. 32, p. 842, 1995

[15] J. M. Corres, I. del Villar, I. R. Matias, and F. J. Arregui, "Two-Layer Nanocoatings in Long-Period Fiber Gratings for Improved Sensitivity of Humidity Sensors," IEEE Trans. Nanotechnol., vol. 7, p. 394, 2008.

[16] B. Gu, M. Yin, A. P. Zhang, J. Qian, and S. He, "Optical fiber relative humidity sensor based on FBG incorporated thin-core fiber modal interferometer," Opt. Express vol. 19, p. 4140, 2011

[17] F. Bueche, "Diffusion of Water in Polymethyl Methacrylate," Journal of Polymer Science, vol.14, p.414, 1954.

[18] J. A. Barrie and B. Platt, "The Diffusion and Clustering of Water Vapour in Polymers," Polymer, vol.4, p.303, 1963.

[19] O. V. Startsev, V. P. Rudnev, and B. V. Perov, "Reversible moisture effects in the climatic ageing of organic glass," Polymer Degradation and Stability, vol.39, pp.373-379, 1993.

[20] A. M. Thomas, "Moisture Permeability, Diffusion and Sorption in Organic Film-Forming Materials," Journal of Applied Chemistry, vol.1, p141, 1951.

[21] T. L. Yeo, T. Sun, K. T. V. Grattan, D. Parry, R. Lade, B. D. Powell, "Polymer-coated fibre Bragg grating for relative humidity sensing," IEEE Sensors J., vol. 5 pp. 1082-1089, 2005.

[22] J. Crank. The Mathematics of Diffusion. $2^{\text {nd }}$ ed., Clarendon Press Oxford, 1975.

[23] D. T. Turner, "Polymethyl methacrylate plus water: sorption kinetics and volumetric changes," Polymer, vol.23, pp.197-202, 1982.

[24] P. Giaccari, H. G. Limberger, and P. Kronenberg, "Influence of humidity and temperature on polyimide-coated fibre Bragg gratings," in Proc. Bragg Gratings, Photosensitivity, and Poling in Glass fibres and Waveguides: Applications and Fundamentals, page BFB2, 2001.

[25] C. Zhang, W. Zhang, D.J. Webb, G.-D. Peng, "Optical fiber temperature and humidity sensor", Electron. Lett., vol.46 p. 643, 2010

[26] G. Wypych, Handbook of solvents. Chem. Tec. Publishing, 2001

[27] K. E. Carroll, C. Zhang, D. J. Webb, K. Kalli, A. Argyros, and M. C. Large, "Thermal response of Bragg gratings in PMMA microstructured optical fibers", Opt. Express, vol. 15, p. 8844, 2007.

[28] W. Yuan, A. Stefani, M. Bache, T. Jacobsen, B. Rose, N. Herholdt-Rasmussen, F. K. Nielsen, S. Andresen, O. B. Sørensen, K. S. Hansen, and O. Bang, " Improved thermal and strain performance of annealed polymer optical fiber Bragg gratings", Opt. Comm., vol. 284 p. 176,2011 